\title{
Hybrid-clustering game Algorithm for Resource Allocation in Macro-Femto HetNet
}

\author{
Fang $\mathrm{Ye}^{1}$, Jing Dai ${ }^{1}$ and Yibing $\mathrm{Li}^{1}$ \\ ${ }^{1}$ College of information and communication engineering, Harbin Engineering University, Harbin, China \\ [e-mail: yefang0923@126.com,jing_d@@163.com, liyibing0920@126.com] \\ *Corresponding author: Yibing Li
}

Received June 15, 2017; revised September 23, 2017; accepted October 8, 2017;

Published April 30, 2018

\begin{abstract}
The heterogeneous network (HetNet) has been one of the key technologies in Long Term Evolution-Advanced (LTE-A) with growing capacity and coverage demands. However, the introduction of femtocells has brought serious co-layer interference and cross-layer interference, which has been a major factor affecting system throughput. It is generally acknowledged that the resource allocation has significant impact on suppressing interference and improving the system performance. In this paper, we propose a hybrid-clustering algorithm based on the Matérn hard-core process (MHP) to restrain two kinds of co-channel interference in the HetNet. As the impracticality of the hexagonal grid model and the homogeneous Poisson point process model whose points distribute completely randomly to establish the system model. The HetNet model based on the MHP is adopted to satisfy the negative correlation distribution of base stations in this paper. Base on the system model, the spectrum sharing problem with restricted spectrum resources is further analyzed. On the basis of location information and the interference relation of base stations, a hybrid clustering method, which takes into accounts the fairness of two types of base stations is firstly proposed. Then, auction mechanism is discussed to achieve the spectrum sharing inside each cluster, avoiding the spectrum resource waste. Through combining the clustering theory and auction mechanism, the proposed novel algorithm can be applied to restrain the cross-layer interference and co-layer interference of HetNet, which has a high density of base stations. Simulation results show that spectral efficiency and system throughput increase to a certain degree.
\end{abstract}

Keywords: Game theory, Clustering, Heterogeneous network, Co-channel interference, Spectrum resource allocation

This paper includes a hybrid-clustering game algorithm based on a Matérn Hard-core Process (MHP) system model. This research was supported by the National Natural Science Foundation of China (Grant No. 51509049), the Natural Science Foundation of Heilongjiang Province, China (Grant No. F201345), the Fundamental Research Funds for the Central Universities of China (Grant No. GK2080260140), the National Key Foundation for Exploring Scientific Instrument of China (Grant No. 2016YFF0102806) and the Provincial Foundation of Natural Science (Grant No. F2017004). 


\section{Introduction}

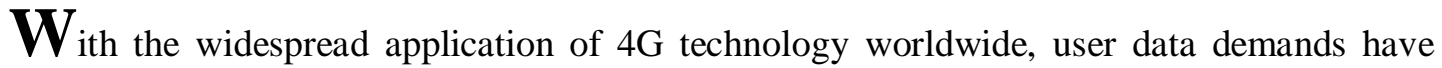
witnessed further increases, causing explosive increases in data quantity in wireless communication systems. According to the 3GPP research report, it is expected that both of the average rate of mobile data business and indoor mobile data transmission business in the following year will exponentially increase [1-3], which becomes a huge challenge for current cellular communication technology to maintain. Femtocells have also been introduced in current cellular networks [4-5] due to their high flexibility and commercialization capability, which can provide better data rates and coverage for indoor users. However, the co-existance of femtocells and macro base stations may create severe co-layer and cross-layer interference. Furthermore, the random nature of femtocells deployment turns the interference into a much more complex issue.

In recent years, resource allocation algorithm is one of the main solutions for the interference suppression problem based on the heterogeneous network (HetNet). For the research of this type of problem, modeling interference for the network is the primary purpose. As the establishment of the interference model needs the location information of the base stations and the users, we firstly adopt a system model, which accurately reflect spatial distribution feature of the base stations. Several literature has discussed this question. System network models of most existing algorithms adopt a regular network model. However, in an actual environment, due to inconsistent capacity demands, instead of being deployed completely based on a regular network model, base stations will display randomness. In terms of modeling cellular networks with irregular base station deployment, Andrews J G. et al. [6] used a homogeneous Poisson point process (HPPP) to model base station distributions in cellular networks, and analyzed two performance indices for coverage and reachable rate. On this basis, Dhillon H S. et al. [7] proposed one type of K-layer cellular network model for heterogeneous cellular network modeling. In this model, each layer of the network consisted of the same type of base station, and each base station layer formed an independent HPPP with their own density. Both of the above references demonstrated that the Poisson point process (PPP) and a typical lattice network could accurately represent the curve of coverage. However, for cellular network station distribution modeling, the homogeneous Poisson point process is not an ideal model because actual base station deployment typically displays a certain repulsion feature. The Matérn hard-core process (MHP) whose points attempt to repel each other acting as a spatial point process can better reflect the characteristic of the actual base station deployment. So, in this paper, considering the repulsion feature of base station deployment in actual network, HetNet model based on MHP is adopted. Furthermore, the positional information of the base stations can be obtained after the system modeled.

In terms of the positional information of the base stations, the interference model can be constructed base on the interference relationships. Necker M C. et al. [8] introduced the interference graph into LTE (Long Term Evolution) macro cellular inter-cell interference coordination, in which the vertex represented users and the edge showed interference between two users. By utilizing a vertex coloring heuristic algorithm, frequency resource blocks were allocated, and two vertices connected by edges could not be allocated to the same block. Based on above, [9-10] presented graph theory to suppress the co-channel interference for downlink transmission in small cell networks. Qiu J. et al. [9] presented a novel clustering approach to establish the interference graph aiming at suppressing interference of ultra-dense small cell 
networks. However, they assumed that some of the vertices, who are named split messengers (SMs), have the high priority to gain the resource in the network. The hypothesis cannot guarantee the fairness of all the users. To ensure the equality of both kind of users, Uygungelen S. et al. [10] adopted both macro users and femto users as the vertices to establish the interference graph. Then used a hybrid vertex coloring algorithm adaptively matched the frequency reuse. However, there is an edge between two vertices is simply defined as a ' 0,1 ' problem, that is, there is an edge as long as there is interference. At the same time, each vertex could been assigned only one channel. It caused the waste of the spectrum resource to a certain extent. Meanwhile, in high-density networks, the signaling overhead for updating the graph is too high due to a complicated interference graph, which then tends to lower overall system throughput capacity. Thus, in this paper, we study the interference modeling based on the system model to solve the questions above. The following part will discuss the interference suppression approach.

Currently, the method of interference suppression based on resource allocation can be divided into three types which includes static, semi static and dynamic. For the static interference suppression method, the wireless resources used by each cell are completely in accordance with the planned distribution scheme. The signaling cost is small but this method is not flexible enough. The semi static interference suppression method is used to adjust the wireless resource of each cell according to the change of load. However, the blocking rate of the edge users is too high to suffer. Compared with the static and semi static interference suppression methods, the dynamic interference suppression method can adjust the user equipment rapidly, therefore the system can achieve the optimal performance [11]. Many scholars use the theory of game to study the dynamic interference suppression based on resource allocation [12-21]. Spectrum resource leasing has become an effective method for improving band and spectrum utilization rate, and is an important technical aspect of heterogeneous network spectrum sharing [12-13]. Frequency spectrum leasing mechanisms that combine economic models such as the auction model have been applied to spectrum sharing in heterogeneous networks [14-17]. Xinbing W. et al. [14] used the auction mechanism for analyzing the division of spectrum resources in cognitive wireless networks. Each cognitive user offered a price for required spectrum resources, and a responsible authorized user network for the spectrum auction decided on spectrum resources to be shared without affecting self-communication quality. The iterations of solutions for cognitive user pricing in distributed cognitive networks is discussed as well. Liang Q. et al. [15] analyzed auction agency based spectrum sharing. In the network, there was a special agency responsible for spectrum resource allocation, which would apply for spectrum resources in a unified manner and then allocate resources at a certain price. Using this type of spectrum resource sharing, information transmission among users is reduced, hence realizing effective sharing of spectrum resources. Hasan N U. et al. [16] presented a novel approach to minimize the interference caused by all secondary users by applying game theory. Furthermore, the method could be found a near-optimal solution with the genetic algorithm. However, the fairness of all the users has not been considered. Hossain E. et al. [17] studied spectrum sharing among cognitive users based on the auction mechanism, and proposed signal-to-interference ratio and power based on the auction mechanism, as well as a Nash equilibrium pricing strategy applicable for iterations of user solutions. However, the above auction mechanism based spectrum sharing model studied centralized spectrum sharing against single or multiple bidders. Besides, majority of studies have focused on the problems, which are based on cognitive wireless networks. Qiu J. et al. [18] introduced a joint power control and frequency resource allocation algorithm. However, only co-tier interference in extremely dense small 
cell networks has been considered. As for a heterogeneous network with relatively large base station density, it is difficult for centralized spectrum resource allocation schemes to realize effective spectrum allocation. As for the resource allocation issue in high-density system networks, clustering theory is typically adopted.

Reference [19-20] considered sub channel allocation for co-channel interference mitigation. For high-density femtocell systems, Li W. et al. [19] used a weighted interference graph to classify each femtocell into disjointing femtocell groups, and then relied on maximal femtocell group capacity to allocate sub-channels for all femtocells. However, this algorithm did not consider the performance improvement of macro base station users. Tang H. et al. [20] proposed one type of hybrid-clustering algorithm, which utilized graph modeling to build an interference graph, and turned channel resource allocation into a hybrid-clustering issue, in which each cluster represented one channel. Different from the conventional coloring algorithm that minimizes color use number to save channel resources, a hybrid-clustering algorithm uses all usable channel resources, with the goal of minimizing system interference to improve user throughput capacity. However, the above method used simple random allocation in reusing spectrum resources, rather than combining effective allocation methods like game theory. Therefore, the key is to build a more reasonable heterogeneous mode, as well as a reasonable system interference gram, and a resource allocation method that reduces cross-layer and co-layer interference.

In this paper, considering the repulsion feature of base stations deployment in the femto tier, we first adopt a heterogeneous network model by applying the Poisson point process with repulsion function. Then, in order to solve the problems in resource allocation algorithms based on game theory, this paper proposes one type of hybrid-clustering game (HCG) algorithm that incorporates equality and improves throughput capacity. The algorithm firstly built a regional average channel state (RACS) based on base station position information to estimate the influence of interference. Signal-to-interference ratio (SINR) is used to judge whether there is a strong interference link. Then considering as well as the interference relationship, an interference graph $G(V, E)$ is built. According to the weights measured by RACS, the problem of optimal clustering partition is evolved into the maximum K-cutting problem in graph theory. Finally, combined with the auction model of game theory, a spectrum sharing model with a single auctioneer and a plurality of bidders is established to allocate the spectrum resources to the users in the same cluster. It makes up for the shortcomings of the traditional clustering algorithm, such as low intermediate frequency band reuse rate and low femtocell throughput. At the same time, it changes the problem of the game theory in the high-density network model, which is difficult to realize the spectrum allocation by using the centralized spectrum resource allocation scheme. The simulation analysis is conducted, and results show that this algorithm can improve spectrum efficiency by introducing a clustering approach to improve resource allocation.

The rest of this paper is organized as follows. Section 2 presents the novel system model. Section 3 introduces the hybrid-clustering game algorithm. In Section 4, simulations are used to evaluate the performance of the proposed algorithm. Finally, we provide a conclusion for the paper

\section{System Model}

\subsection{System Model}


Consider a bilayer downlink HetNet that consists of a macro base station $M B S_{M}$ and several femtocells $F B S_{j}, j \in\{1,2, \ldots, N\}$, as shown in Fig. 1, the solid line represents the usable signal, while the dashed line represents the interference signal.

The usual method for modeling cellular networks is HPPP, which reflect the random nature of base station distribution to a certain extent. However, using HPPP to model the base station position in a cellular network means that all deployed base stations are completely independent. Actually, each base station has a certain coverage scope and overlapping coverage bringing complex interference. Thus, base station placement will display a certain repulsion feature. In order to reflect the negative correlation of the base station location more accurately, in this paper, spatial points with a repulsion function among nodes are adopted to model the heterogeneous network.

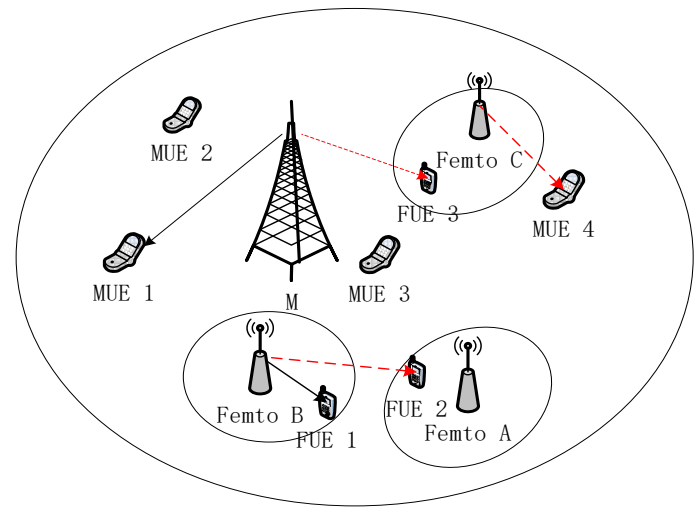

Fig. 1. Downlink transmission links in HetNet

We assume that base stations of the same type be in the same layer and have the same transmission power. Moreover, the same layer base station distribution conforms to one HPPP $\Phi_{j}$ with a density $\lambda_{j}$ (see Fig. 2(a)). Then, based on the repulsion radius $r_{h}$, we thin $\Phi_{j}$ to obtain $\hat{\Phi}_{j}$. Firstly, we assign a random value uniformly distributed in $[0,1]$ to each point in $\Phi_{j}$. Then we take any point in $\Phi_{j}$ and take the point as the center of the circle $r_{h}$ radius. We delete all points in the circle that coexist within a distance less than $r_{h}$ from the point with a lower value. Hence, only points with the lowest value in their $r_{h}$ neighborhood distance are retained. The repulsion radius $r_{h}$ is the actual radius of the base station [22]. In this manner, iterate through every point in $\Phi_{j}$ on this account. After being thinned, the obtained spatial point process $\hat{\Phi}_{j}$ becomes a Matérn Hard-core Process (MHP) and the points in $\hat{\Phi}_{j}$ are the centers of hard balls of radius $r_{h} / 2$. The density $\hat{\lambda}_{j}$ of this spatial point process can be obtained by equation (1).

$$
\hat{\lambda}_{j}=\frac{1-\exp \left(-\lambda_{j} \pi r_{h}^{2}\right)}{\pi r_{h}^{2}}
$$


Fig. 2(b) is a HetNet model built in this paper based on MHP. The coverage of each base station does not overlap, and the location relationship among base stations is negatively correlated. Meanwhile, due to the dilution of the femtocell distribution, the interference between the base station and the user is simplified, and the complexity of the system is reduced.

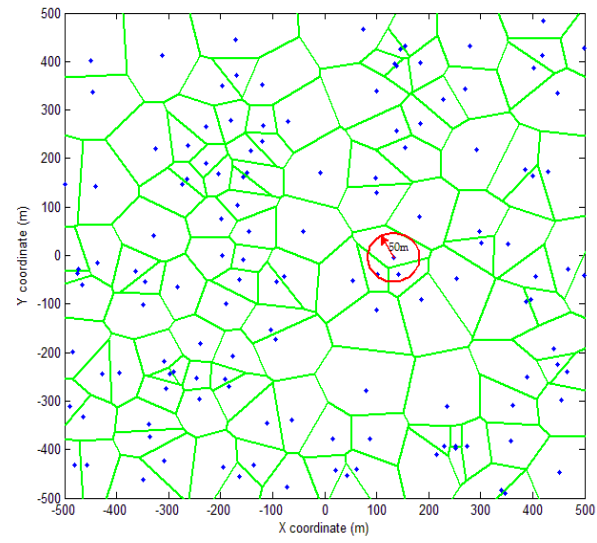

(a) Poisson point process $\Phi_{j}$ with density $\lambda_{j}$

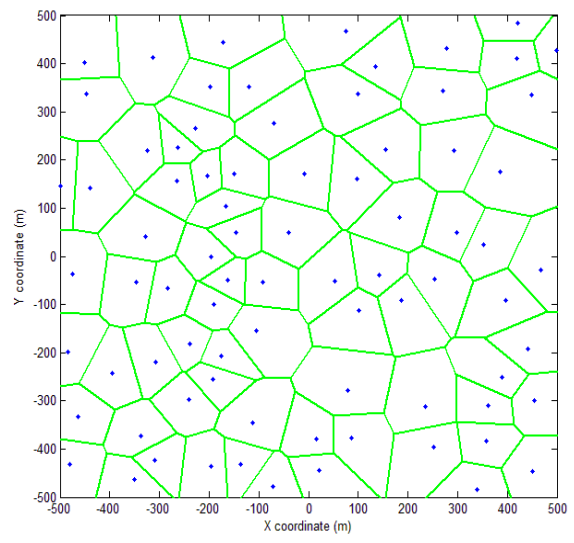

(b) MHP $\hat{\Phi}_{j}$ with density $\hat{\lambda}_{j}$ thinned from $\Phi_{j}$ Fig. 2. The location of femtocells based on PPP

\subsection{Interference Model}

Based on the base station position information in Fig. 2(b) and the system interference relationship, the interference graph $G(V, E)$ is developed as shown in Fig. 3, where $V=\left\{V_{1}, V_{2} \ldots\right\}$ represents vertices (shown as circles) and $E=\left\{E_{1}, E_{2} \ldots\right\}$ represents edges (shown as straight lines).

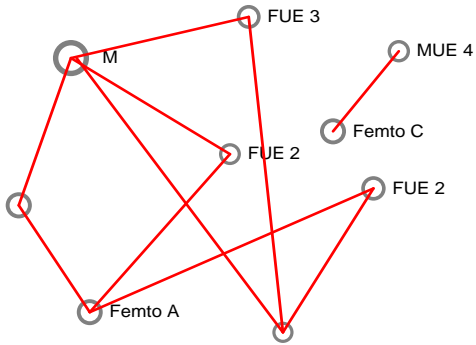

Fig. 3. Schematic plot of interference graph

In a traditional interference graph, the vertex normally only contains femtocell users or macro base station users. Besides, whether there is an edge exist between two vertices is simply defined as a ' 0,1 ' model. That is, the edge exists is identified ' 1 ' as long as the transmission link is an interference link. Otherwise, the edge does not exist and is identified as ' 0 '. This type of interference graph cannot ensure equality. Meanwhile, in high-density networks, the signaling overhead for updating the graph is too high due to the complicated interference graph, which then tends to lower overall system throughput capacity. In order to ensure equality among different base station users, we choose all femtocell and macro base station community users as vertices in the graph and manage femtocells at an equal level. 
Moreover, we propose the strong interference indication matrix $C=\left\{C_{i j} \mid C_{i j}=0,1\right\}$ to improve the method for judging the existence of edge. Only if there is a strong interference link between two vertices based on signal-to-interference ratio, can the edges of the two vertices be considered to exist. That is, when $C_{i j}=1$, the interference between vertices is judged large enough, and it is considered as a strong interference link. For better assessment of interference, a regional average channel state $(R A C S)$ is built to estimate the influence of interference. $R A C S$ is the threshold used to determine whether the value of $C_{i j}$ is defined as $C_{i j}=1$. When $C_{i j}=1$, the vertex interference is large and there exists a strong interference link; That is, when $R A C S<S I N R_{t h}=c_{t h}$, there should be an edge representing the interference between two vertices. $c_{t h}$ is a predefined threshold [23]. RACS of region $m$ served by $F_{i}$ and interfered by $F_{j}(i \in V, j \in V)$ represents the average signal-to-interference plus noise ratio, which is calculated based on the location information of the base station:

$$
\begin{array}{r}
\operatorname{SINR}_{i, j}(x, y)=P_{r, i}(x, y) /\left(P_{r, j}(x, y)+N_{0}\right) \\
R A C S\left(i, j, A_{i}\right)=\iint_{A_{m}} \operatorname{SINR}_{i, j}(x, y) d x d y / S\left(A_{i}\right)
\end{array}
$$

where $i \in V, j \in V, i \neq j ; P_{r, i}$ is the signal power sent from base station $B S_{i}$ and received by user $r ; N_{0}$ is noise power; $S\left(A_{i}\right)$ is the size of $A_{i}$, and $A_{i}$ is the coverage area of base station $B S_{i}$.

Three different interference scenarios are analyzed with the use of regional average channel state, and three types of interference between the vertices discussed above are as follows.

1. Interference between different femtocells.

Choose femtocells $F B S_{i}$ and $F B S_{j}$ located at $(0,0)$ and $(d, 0)$, respectively, with base station radii $R_{i}$ and $R_{j} . P_{i}$ and $P_{j}$ represent the transmit power of $F B S_{i}$ and $F B S_{j}$, respectively. $R A C S_{\left(i, j, A_{i}\right)}$ indicates the average channel quality of the $F B S_{i}$ coverage area under the interference $F B S_{j}$.

$$
\operatorname{RACS}_{\left(i, j, A_{i}\right)}=\iint_{A_{i}} \frac{P_{r, i}(x, y)}{P_{r, j}(x, y)+N_{0}} d x d y / S\left(A_{i}\right)
$$

Path loss mode uses the indoor transmission formula $L_{c}($ dis $)=128+37.6 \log _{10}($ dis $)$, where dis indicates the distance between the $F B S_{i}$ user and base station. Ignoring background noise, $\operatorname{RACS}_{\left(i, j, A_{i}\right)}$ can be approximated as follows: 


$$
\operatorname{RACS}_{\left(i, j, A_{i}\right)} \approx \frac{P_{i}}{\pi P_{j}\left(R_{i}^{2}-R_{\min }^{2}\right)} \int_{0}^{2 \pi} \int_{R_{\min }}^{R_{i}}\left(\frac{r^{2}+d^{2}-2 r d \cos \theta}{r^{2}}\right)^{1.5} r d r d \theta
$$

where $R_{\min }$ is the shortest distance between the femtocell user and femtocell.

2. Interference of macro base station on femtocell.

The same schematic details as provided in 1 are used. Assuming one base station $M B S_{M}$ located at $(D, 0)$ with transmission power $P_{M}$. Since the macro base station covers the whole community, $F B S_{k}$ femtocell users will be interfered by macro base station $M B S_{M}$. Similar to scenario one, in the path loss model, the influence of the macro base station on the femtocell uses the urban space transmission formula $L_{c}($ dis $)$, and users from the femtocell to the base station coverage area use the indoor transmission formula $127+30 \log _{10}($ dis $)$. Ignoring background noise, we can approximate obtain:

$$
\operatorname{RACS}_{\left(i, M, A_{i}\right)} \approx \frac{P_{i}}{P_{M}} \cdot \frac{2}{R_{i}+R_{\min }}\left(\frac{1}{3}\left(R_{i}^{2}+R_{\min }^{2}+R_{i} R_{\min }\right)+\frac{D^{4}}{R_{i} R_{\min }}+4 D^{2}\right)
$$

3. Interference of femtocell on macro base station users.

In this scenario, assuming one macro base station $M B S_{M}$ with transmission power $P_{M}$ and one femtocell $F B S_{k}$ located at $\left(x_{f}, y_{f}\right)$ with transmission power $P_{F}$. When macro base station user $M U E_{i}$ is located at $(x, y)$, ignoring noise power and using the urban space transmission formula $L_{c}($ dis $)$, we can approximate as follows:

$$
\operatorname{RACS}_{\left(M, i, A_{i}\right)} \approx \frac{P_{i}}{\pi P_{M} D^{2}} \int_{0}^{2 \pi} \int_{R_{\min }}^{R_{M}}\left(\frac{r^{2}+r_{f}^{2}-2 r r_{f}}{r^{2}}\right)^{1.88} r d r d \theta
$$

Distinguishing the existence of strong interference between two vertices by $R A C S$, we build strong interference indication matrix $C=\left\{C_{i j} \mid C_{i j}=0,1\right\}$. When the existence of the edge of all vertices is determined, we obtain an accurate interference graph. RACS is also used to analyze interference weight in the clustering scheme which is elaborated in the next section.

In the next section, we transform the problem of channel resource allocation into the problem of hybrid clustering according to the interference graph. The objective is to minimize the interference in order to improve throughput. The auction mechanism is used to allocate the spectrum resources to the users in the same cluster in order to achieve the reasonable allocation of the spectrum. 


\section{Game Model and Problem Statement}

\subsection{Hybrid Clustering Algorithm}

The HCG algorithm is divided into two parts. First, we propose a hybrid-Dsatur clustering algorithm to minimize co-channel interference. We assume that the number of sub-bands is $K$, the same node can be assigned to different clusters, and each node can reuse multiple channels. In order to improve throughput, the problem is formulated as follows:

$$
\begin{array}{r}
\arg \max \sum_{k=1}^{K} \sum_{i=1}^{N} B_{k} \log _{2}\left(1+\operatorname{SINR}_{k} \delta_{k, i}\right) \\
\text { subject to }: \delta_{k, i}=1 \text { or } 0, i \in V k \in[1, K] \\
B_{k} \log _{2}\left(1+\operatorname{SINR}_{k} \delta_{k, i}\right) \geq R^{\min }
\end{array}
$$

where $N$ is the total number of vertices $N=|V|, B_{k}$ is a sub-band marked $k, k \in[1, K]$, and sub-band $k$ will assign to node $i, i \in V$ when $\delta_{k, i}=1$, otherwise $\delta_{k, i}=0 . R^{\min }$ is the minimum rate requirement of users.

To determine the possibility that vertices belong to the same cluster, the interference weights between vertice $i$ and vertice $j$ is defined as $c c_{i j}$. The graph interference weights matrix $C C=\left[c c_{i j}\right]_{N \times N}, i \in V, j \in V$ can be constructed. Since two macro users cannot be assigned to the same sub-band, weight between which can be set as $c c_{0}$ (a very large value). Meanwhile, we consider a macro user with higher priority than the femto user. $c c_{0}$ is also assigned to denote interference from the femtocell to the macro user in order to ensure the performance of the macro user. For other types of interference, 1/RACS is used to express the amount of interference. The purpose of this algorithm is to assign all the elements of the vertice set $V$ to $S$ orthogonal sets by using the interference weight matrix. Hence, the channel assignment problem can be described as a clustering process. In regular clustering schemes, the common target is to minimize the number of clusters. However, our proposed clustering scheme uses all available channels and aims to minimize interference in order to improve throughput.

The $K$ resource blocks are divided into $S$ segments, and each segment contains $R$ resource blocks. Each spectrum resource represents a cluster, which defines the user cluster number group $S$ as $[1,2, \ldots, S]$. Vertices are divided into the same cluster and share spectrum resources. In order to partition the vertices into $S$ clusters, we first calculate the sum interference $s u m_{-} c c_{i}$, which expresses the total interference of node $i$ suffered from others. the parameter is calculated as follows:

$$
s u m_{-} c c_{i}=\sum_{j=1}^{N} c c_{i j}, i \in[1, N]
$$


Then, we mark the nodes ordered by the value of $s u m_{-} c c_{i}$. The node with the lowest value of sum $\_c c_{i}$ will first be assigned into clusters so that we minimize interference on the graph. In order to improve spectrum efficiency, the process of assigning nodes into clusters is expressed as three steps:

Step 1: In order to ensure the communication quality of macro users, $S$ macro users with the highest level of spectrum requirements are assigned to the clusters at first. These users with higher communication quality requirements become cluster heads. Each cluster is traversed until each cluster has a cluster head. Then proceeding to allocate other clusters.

Step 2: Dividing the remaining users into different clusters as far as possible to meet the minimum service guarantee requirements. We judge whether node $i$ can be assigned into cluster $k(k \leq S)$ according to the $n^{\text {th }}$ number of the cluster. Three conditions must be satisfied:

a. All nodes of $c_{j i}, j \in \mathbb{M}_{k}$, meet $c_{j i}=0, \forall j \in \mathbb{M}_{k}$, where $\mathbb{M}_{k}$ is the current node set of cluster $k$.

b. $\quad c c_{-} l s_{k}<1 / S I N R_{t h}$, where $c c_{-} l s_{k}$ is the sum interference of node $i$ as suffered from all current nodes in cluster $k . c c_{-} l s_{k}$ is calculated as:.

$$
c c_{-} l s=\sum_{j=1}^{n} c c_{\text {cluster }_{(k, j)}} i ;\left|\mathbb{M}_{k}\right|=n
$$

c. price_cc $c_{j}<1 / \operatorname{SINR}_{t h}, \forall j \in \mathbb{M}_{k}$, where price_cc ${ }_{j}$ is the sum interference of current node $j$ if node $i$ is assigned into cluster $k$. price $\_c c_{j}$ is calculated as:

$$
\text { price_cc } c_{k}=\sum_{j=1}^{n} c c_{\text {cluster }(m, j)} k, j \neq k ;\left|\mathbb{M}_{k}\right|=n
$$

Once being assigned into a cluster, node $i$ will traverse all clusters until all conditions are met. We then allocate the node into cluster $k$, which has the minimum $c c_{-} l s_{k}$ : $\min \left(c c_{-} l s_{k}\right)$, if there is no appropriate cluster for a node. This will ensure network fairness. The second step assigns nodes into different clusters in order to guarantee that each node can reuse a sub-band.

Step 3: In order to improve throughput and spectrum efficiency, the same node can be assigned to different clusters. In the third step, we repeat the second step for each node. The clustering process stops when no node can be assigned into a cluster.

Since the number of clusters is guaranteed to be the same as the number of sub spectrum band. therefore, one band is allocated for each cluster, and the same frequency band is used by all users in the same cluster. Based on the clustering results, $S$ section spectrum resources will be randomly allocated to clusters. Spectral efficiency will be enhanced because the same user can reuse multiple channels. By using the auction mechanism, spectrum resources obtained by the cluster are distributed to each cluster node based on spectrum bidding of nodes in the cluster. 


\subsection{Auction Mechanism Based Spectrum Sharing}

An auction mechanism based spectrum sharing model can solve spectrum resource sharing issues in the case of one single clustering node or multiple clustering nodes. A clustering node has priority for spectrum resources and the pricing right for spectrum resource leasing and allocation. In order to ensure the communication speed of clustering node user, a clustering node can lease out and allocate spectrum resources to nodes inside the cluster, hence meeting the communication requirements of clustering nodes and obtaining economic revenue. Therefore, spectrum resource sharing can be considered as a spectrum resource auctioning model. We consider the auction model has one primary user and a group of secondary users. The primary user is the cluster head who will share the frequency resource with the other nodes in the cluster who acting as secondary users. As all the nodes are selfishly to maximize its own utility, we consider the auction is a non-cooperative game. Nodes in the cluster can apply for spectrum resources from cluster head nodes through spectrum resource biding. The cluster head node is the auctioneer of spectrum resources and is responsible for collecting offers from nodes and determining the size of spectrum resources to be allocated.

Assuming that the revenue obtained by a unit occupying spectrum resources is known for nodes in the cluster, and the maximal transmission speed obtained by nodes in the cluster is regarded as the revenue obtained by nodes in obtaining the use right [24]. For that reason, we define the utility function as the transmission speed obtained by nodes.

Nodes in the cluster submit spectrum resource prices based on self-communication demand. Assuming that $\boldsymbol{b}^{\prime}=\left[b_{1}^{\prime}, b_{2}^{\prime}, \ldots, b_{N_{c}}^{\prime}\right]$ is the price offered by nodes in the cluster and that $N_{c}$ is the number of nodes in the cluster. Reference [24] regarded spectrum resource prices as the resource sizes applying to be occupied. $0 \leq b_{i}^{\prime} \leq B^{\text {tot }}$, where $B^{w}$ is the spectrum resource bandwidth of nodes in the cluster. As the spectrum auctioneer, cluster head nodes collect spectrum resource prices $\boldsymbol{b}^{\prime}$ from cluster nodes and determine the shared sizes $\boldsymbol{B}=\left[B_{1}, B_{2}, \ldots, B_{N_{c}}\right]$ based on cluster nodes' possessed spectrum resources and the condition of ensuring sufficient resources for itself,

$$
B_{i}=\frac{b_{i}^{\prime}}{\sum_{i=1}^{N_{c}} b_{i}^{\prime}+\alpha} B^{w}
$$

where $\alpha$ is the ratio of spectrum resources retained by the cluster head node. In addition, given that the cluster head node needs to ensure its own communication quality, shared spectrum resources $\boldsymbol{B}$ provided to cluster nodes have to satisfy $B^{w}-\sum_{i=1}^{N_{c}} B_{i}>B^{r}$, where $B^{r}$ are the spectrum resources required by the cluster head node to ensure self communication quality. The cluster head node will lease out remaining idle spectrum resources to cluster nodes at unit price $u$.

The earnings $R_{i}$ obtained by node $i$ in the cluster due to shared spectrum resources provided by the cluster head node are:

$$
R_{i}=r_{i} B_{i}
$$


where $r_{i}$ is the revenue obtained through unit transmission speed.

The cost $C_{i}$ for node $i$ in the cluster to obtain shared spectrum resources $B_{i}$ is:

$$
C_{i}=u l_{i} b_{i}^{\prime}
$$

where $l_{i}$ is the priority factor of the cluster node.

Therefore, the revenue function for node $i$ to apply for spectrum resources at the offered price $b_{i}^{\prime}$ is:

$$
\begin{aligned}
U_{i}\left(b_{i}^{\prime}, \boldsymbol{b}_{-i}^{\prime}, u\right) & =R_{i}\left(B_{i}\left(b_{i}^{\prime}, \boldsymbol{b}_{-i}^{\prime}\right)\right)-C_{i}\left(b_{i}^{\prime}, u\right) \\
& =r_{i} \frac{b_{i}^{\prime}}{\sum_{i=1}^{N_{c}} b_{i}^{\prime}+\alpha} B^{w}-u l_{i} b_{i}^{\prime}
\end{aligned}
$$

where $\boldsymbol{b}_{-i}^{\prime}=\left[b_{1}^{\prime}, b_{2}^{\prime}, \ldots, b_{i-1}^{\prime}, b_{i+1}^{\prime}, \ldots, b_{N_{c}-1}^{\prime}\right]$ is the price offered by each node except node $i$.

Nodes in the cluster choose a spectrum offer price $\left(b_{i}^{\prime}\right)^{*}$ that maximizes their revenue function according to their own communication parameters to achieve Nash equilibrium. Nodes in the cluster cannot obtain a higher spectrum revenue by changing the pricing strategy; namely, the spectrum offer price for each node has to satisfy $\left(b_{i}^{\prime}\right)^{*}$ :

$$
\left(b_{i}^{\prime}\right)^{*}=\underset{0 \leq b_{i}^{\prime} \leq B^{w}}{\arg \max } U_{i}\left(b_{i}^{\prime}, \boldsymbol{b}_{-i}^{\prime}, u\right)
$$

where $\left(\boldsymbol{b}^{\prime}\right)^{*}=\left[\left(b^{\prime}\right)_{i}^{*},\left(\boldsymbol{b}_{-i}^{\prime}\right)^{*}\right]$. In order to find the spectrum price offer strategy that maximizes the shared spectrum revenue for cluster nodes, let $\partial U_{i}\left(b_{i}^{\prime}, \boldsymbol{b}_{-i}^{\prime}, u\right) / \partial b_{i}^{\prime}=0$. Then, the following can be obtained:

$$
r_{i} B^{w} \frac{\sum_{j=1, j \neq i}^{N_{c}} b_{j}^{\prime}+\alpha}{\left(\sum_{j=1}^{N_{c}} b_{j}^{\prime}+\alpha\right)^{2}}-u l_{i}=0
$$

Since node $i$ does not know the offer price of other nodes, the solution is similar to the sharing spectrum price in the competitive price game model through obtaining the spectrum offer price of nodes through multiple iterations

$$
\left(b_{i}^{\prime}\right)^{t+1}=\left(b_{i}^{\prime}\right)^{t}+\delta_{i}\left(b_{i}^{\prime}\right)^{t} r_{i} B^{w} \frac{\sum_{j=1, j \neq i}^{N_{c}} b_{j}^{\prime}+\alpha}{\left(\sum_{j=1}^{N_{c}} b_{j}^{\prime}+\alpha\right)^{2}}-\delta_{i}\left(b_{i}^{\prime}\right)^{t} u l_{i}
$$

where $\left(b_{i}^{\prime}\right)^{t}$ is the spectrum offer price of node $i$ at moment $t$. 


\section{Simulation Results and Analysis}

In this section, we conduct simulations in order to assess algorithm performance. First, we analyze the auction mechanism based spectrum sharing model in one cluster. Then, we simulate and analyze throughput of the HCG algorithm. Simulation parameters are shown in table 1.

Table 1. Simulation parameters

\begin{tabular}{|c|c|c|}
\hline Parameter & Macrocell & Femtocell \\
\hline \hline System Bandwidth & $20 \mathrm{MHz}$ & $20 \mathrm{MHz}$ \\
\hline Cell Radius & $500 \mathrm{~m}$ & $50 \mathrm{~m}$ \\
\hline Antenna Gain & $14 \mathrm{dBi}$ & $0 \mathrm{dBi}$ \\
\hline Transmit Power & $46 \mathrm{dBm}$ & $21 \mathrm{dBm}$ \\
\hline User Distribution & 90 per cell & 2 per cell \\
\hline$c c_{0}$ & 10000 & 10000 \\
\hline$c_{t h}$ & $10 \mathrm{~dB}$ & $10 \mathrm{~dB}$ \\
\hline
\end{tabular}

Assuming that the cluster head node possesses a section of spectrum resource, we consider that the same type of vertices rent the spectrum at the same price. Nodes in the cluster share spectrum resources of the cluster head node to obtain a certain transmission speed; per unit of transmission speed can obtain the spectrum revenue $r_{i}=10$; per unit price for the cluster head node provides bandwidth for sharing $u=10$.

Fig. 4 shows a simulation analysis where nodes in the cluster quickly obtain a stable spectrum offer price strategy by an iterative solution through adopting the auction mechanism based spectrum sharing model. Clustering theory based spectrum allocation models do not analyze how to further allocate spectrum resources to nodes in the cluster. While auction mechanism based spectrum leasing models mainly discuss the spectrum sharing issue between single spectrum resource providers and multiple nodes in the cluster, and the determination of spectrum resources to be shared by the auctioneer through collection of spectrum offer prices. Therefore, first we use clustering theory to simplify the complicated interference topological structure, treat cluster head nodes as the spectrum auctioneers, and use the auction mechanism to complete spectrum resource sharing among cluster nodes.

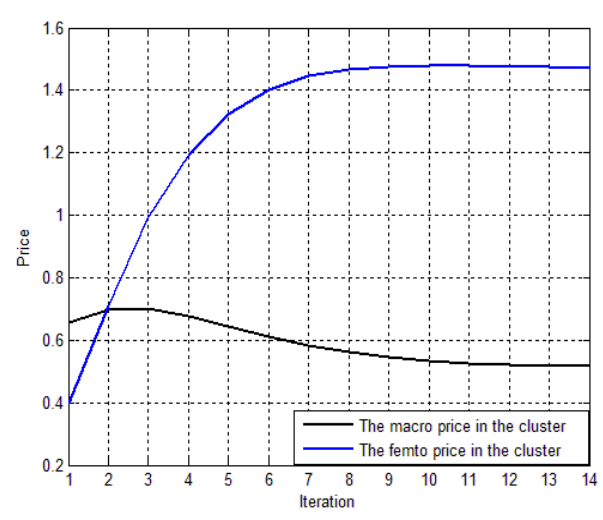

Fig. 4. Spectrum offer price strategy

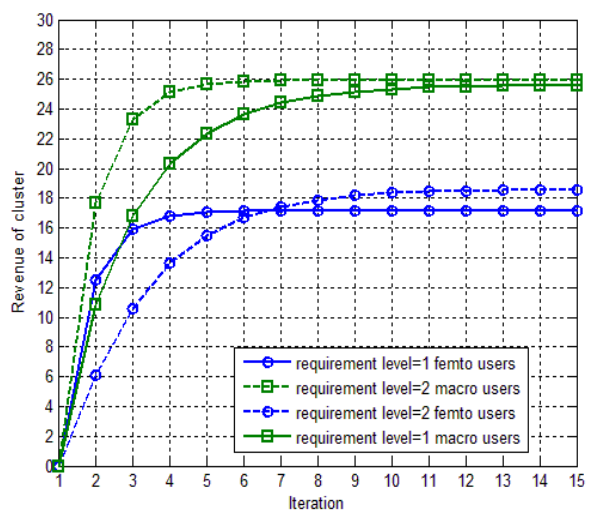

Fig. 5. Revenue obtained for different requirement levels 
Fig. 5 shows the revenue obtained by each cluster head node by providing nodes in the cluster with spectrum resources for different requirements. Simulation results show that with the increase of requirement level, nodes in the cluster tend to apply for more shared spectrum resources from cluster head through the requirement level factor, and generate higher sharing spectrum revenue for cluster head. When this spectrum leasing mechanism, which considers the requirement of nodes in the cluster, is applied to clustering game spectrum sharing, all cluster heads consider the influence of spectrum resource requirements at each node while conducting resource leasing and sharing, which is more similar to an actual application.

Fig. 6 (a) shows channel average multiplexing curves for various algorithms with different macro user density. Simulation results show that the proposed algorithm is more efficient than other algorithms. In addition, with an increase in the number of macro users, the average number of HCG channels decreases. HCIG allocates only one channel for users in the network, as well as the network channel number reuse time sufficient for all users, so, with an increase in the number of macro users, the number of the corresponding channel multiplexing is also increased. Fig. 6 (b) shows channel average multiplexing curves for various algorithms with different femtocell density. Simulation results show that HCG is more efficient than existing algorithms. With the increase of the number of femtocells, the average number of channel multiplexing for HCG increases because HCG is based on the principle of maximizing throughput allocation and is bound to allocate more channels for the femtocell. This reduces total network throughput for macro user services and result in an increase in the number of channel multiplexing. At the same time, HCG ensures the communication quality requirements of users, and in this macro set the macro users are unchanged. The number of multiplexed channels does not change much, meaning that the influence of the macro base station on allocation of network spectrum resources remains basically unchanged. Therefore, users with higher communication quality requirements are generally macro users.

Fig. 7 shows average throughput curves for different algorithms as the increase of femto users. The overall performance of the proposed algorithm is better than other algorithms due to the increase in HCG channel multiplexing intensity, which enables users to get more channels for communication. With an increase in macro station users, the channel number assigned to the femtocell decreased, so the overall trend is downward.

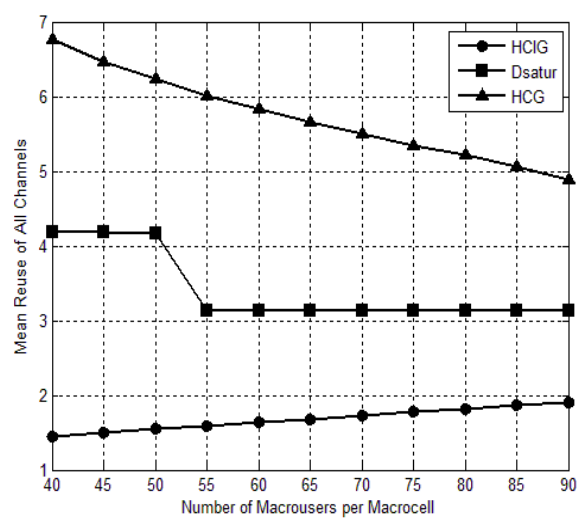

(a) all users as macro users increases

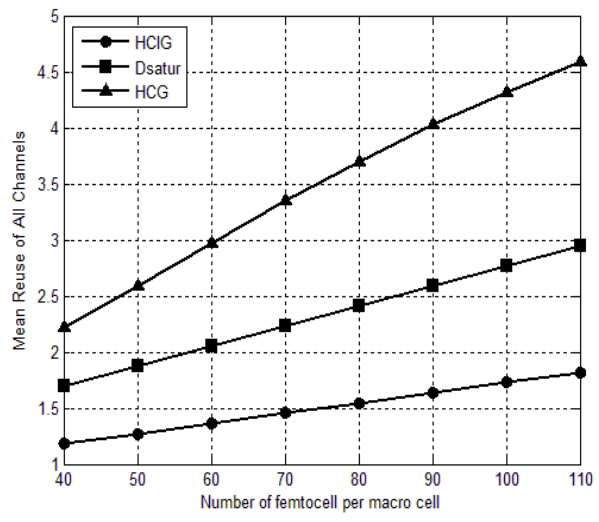

(b) all users as femtocell increases

Fig. 6. Channel average curves 


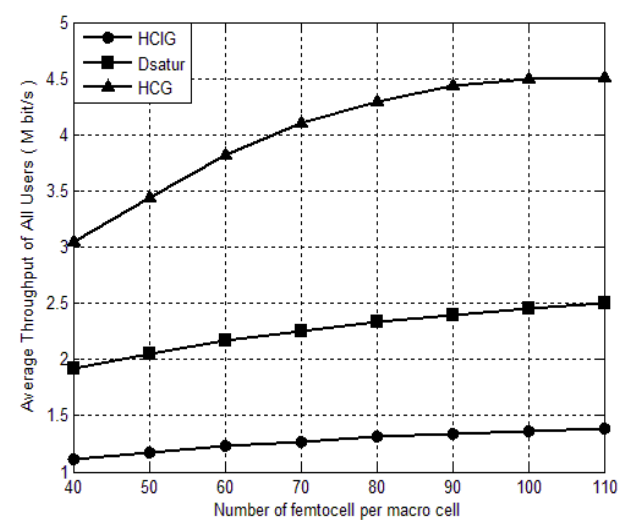

(a) Average throughput curves of all users as femto users increases

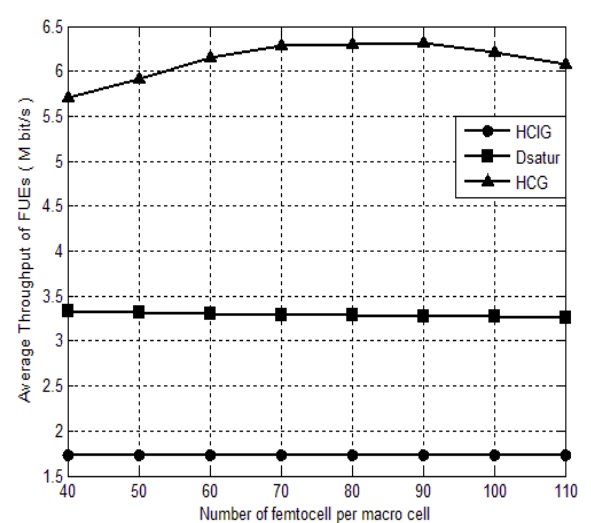

(b) Average throughput curves of femto users as femto users increases

Fig. 7. Average throughput curves

\section{Conclusion}

In this paper, a network model with random characteristics and a negative correlation between base stations is established by MHP to solve the problem of heterogeneous networks with a high density of base stations. The improved system model can be used to accurately model interference in heterogeneous networks. We introduce Regional Average Channel State based on the location interference of base stations to minimize interference. Cluster theory is introduced to make small interfering peaks in the interference graph share the same spectrum resources. In order to suppress cross-layer and co-layer interference, femtocells and macro users are treated on the same level. Combined with the auction model of game theory, the spectrum leasing mechanism is an effective method to improve frequency spectrum utilization. In order to resolve this problem, existing algorithms mainly study the spectrum sharing model with a single auctioneer and a plurality of bidders. The proposed algorithm improves spectral efficiency by introducing an improved auction mechanism as well as a new clustering scheme.

\section{Acknowledgement}

This paper was supported by the National Natural Science Foundation of China (Grant No. 51509049), the Natural Science Foundation of Heilongjiang Province, China (Grant No. F201345), the Fundamental Research Funds for the Central Universities of China (Grant No. GK2080260140), the National Key Foundation for Exploring Scientific Instrument of China (Grant No. 2016YFF0102806), and the Provincial Foundation of Natural Science (Grant No. F2017004).

\section{References}

[1] 3GPP, "3rd Generation Partnership Project; Technical Specification Group Services and System Aspects; Feasibility study for proximity services (ProSe) (Release 12),” TR 22.803, V12.2.0, Jun. 2013. Article (CrossRef Link) 
[2] Bouras C, Diles G, Kokkinos V, et al, "Transmission optimizing on dense femtocell deployments in 5G," International Journal of Communication Systems, vol.29, no.16, pp.2388-2402, 2015. Article (CrossRef Link)

[3] Elsawy H, Hossain E, Haenggi M, "Stochastic Geometry for Modeling, Analysis, and Design of Multi-Tier and Cognitive Cellular Wireless Networks: A Survey," Communications Surveys \& Tutorials IEEE, vol.15, no.3, pp.996-1019, 2013. Article (CrossRef Link)

[4] Boccardi F, Heath R W, Lozano A, et al, "Five disruptive technology directions for 5G," Communications Magazine IEEE, vol.52, no.2, pp.74-80, 2014. Article (CrossRef Link)

[5] Jo M, Maksymyuk T, Batista R L, et al. "A survey of converging solutions for heterogeneous mobile networks," IEEE Wireless Communications, vol.21, no.6, pp.54-62, 2014.

Article (CrossRef Link)

[6] Andrews J G, Baccelli F, Ganti R K, "A Tractable Approach to Coverage and Rate in Cellular Networks," IEEE Transactions on Communications, vol.59, no.11, pp.3122-3134, 2011. Article (CrossRef Link)

[7] Dhillon H S, Ganti R K, "Modeling and Analysis of K-Tier Downlink Heterogeneous Cellular Networks," IEEE Journal on Selected Areas in Communications, vol.30, no.3, pp.550-560, 2012. Article (CrossRef Link)

[8] Necker M C, "Scheduling Constraints and Interference Graph Properties for Graph-based Interference Coordination in Cellular OFDMA Networks," Mobile Networks and Applications, vol.14, no,4, pp.539-550, 2009. Article (CrossRef Link)

[9] Qiu J, Wu Q, Xu Y, et al. "Demand-aware resource allocation for ultra-dense small cell networks: an interference-separation clustering-based solution," Transactions on Emerging Telecommunications Technologies, vol.27, no.8, pp. 1071-1086, 2016. Article (CrossRef Link)

[10] Uygungelen S, Auer G, Bharucha Z, "Graph-Based Dynamic Frequency Reuse in Femtocell Networks," IEEE Vehicular Technology Conference (VTC Spring), pp.1-6, 2011.

Article (CrossRef Link)

[11] Ahmad I, Liu S, Feng Z, et al, "Game Theoretic Approach for Joint Resource Allocation in Spectrum Sharing Femtocell Networks," Journal of Communications \& Networks, vol.16, no.6, pp. 627-638, 2014. Article (CrossRef Link)

[12] Fu J, Xiong S, Liu W, et al. "A new power control algorithm based on game theory in Cognitive Radio system," in Proc. of International Conference on Advanced INFOCOM Technology IET, pp.1-5, 2012. Article (CrossRef Link)

[13] He Q, Zhu L, Mao H, "A new spectrum allocation algorithm based on game theory in cognitive radio networks," Inderscience Publishers, vol.21, no.2, pp.82-88, 2016. Article (CrossRef Link)

[14] Xinbing W, Zheng L, Pengchao X, Youyun X, et al, "Spectrum Sharing in cognitive radio networks: An auction-based approach," IEEE Transactions on Systems, Man, and Cybernetics, Part B: Cybernetics, vol.40, no.3, pp. 587-596, 2010. Article (CrossRef Link)

[15] Liang Q, Feng Y, Lin G, Xiaoying G, et al, "Spectrum trading in cognitive radio networks: An agent-based model under demand uncertainty," IEEE Transactions on Communications, vol.59, no.11, pp. 3192-3203, 2011. Article (CrossRef Link)

[16] Hasan N U, Ejaz W, Ejaz N, et al. "Network Selection and Channel Allocation for Spectrum Sharing in 5G Heterogeneous Networks," IEEE Access, vol. 4, pp. 980-992, 2016.

Article (CrossRef Link)

[17] Zhu K, Hossain E, Niyato D, "Pricing, Spectrum Sharing, and Service Selection In two-tier Small Cell Networks: A Hierarchical Dynamic Game Approach," IEEE Transactions on Mobile Computing, vol.13, no.8, pp. 1843-1856, 2014. Article (CrossRef Link) 
[18] Qiu J, Ding G, Wu Q, et al. "Hierarchical Resource Allocation Framework for Hyper-Dense Small Cell Networks," IEEE Access, vol.4, no.99, pp. 8657-8669, 2016. Article (CrossRef Link)

[19] Li W, Su T, Zheng W, "Dynamic Clustering Based Sub-Band Allocation in Dense Femtocell Environments," in Proc. of IEEE Vehicular Technology Conference (VTC Spring), pp.1-5, 2012. Article (CrossRef Link)

[20] Tang H, Hong P, Xue K, Peng J, "Cluster-Based Resource Allocation for Interference Mitigation in LTE Heterogeneous Networks," in Proc. of IEEE Vehicular Technology Conference (VTC Spring), pp.3-6, 2012. Article (CrossRef Link)

[21] Han S, Li X, Liu Z, "Hierarchical-game-based algorithm for downlink joint subchannel and power allocation in OFDMA femtocell networks," Journal of Network and Computer Applications, vol.73, pp. 44-56, 2016.

[22] Dhillon H S, Ganti R K, Andrews J G, "Load-Aware Modeling and Analysis of Heterogeneous Cellular Networks," IEEE Transactions on Wireless Communications, vol.12, no.4, pp.1666-1677, 2013. Article (CrossRef Link)

[23] Cho S, Choi W, "Coverage and Load Balancing in Heterogeneous Cellular Networks with Minimum Cell Separation," IEEE Transactions on Mobile Computing, vol.13, no.9, pp.955-1966, 2014. Article (CrossRef Link)

[24] Parvin S, Hussain F K, Hussain O K, et al. "Cognitive radio network security: A survey," Journal of Network \& Computer Applications, vol.35, no.6, pp.1691-1708, 2012. Article (CrossRef Link)

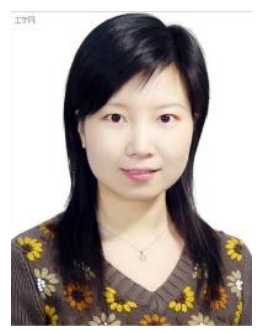

Fang Ye received the B.S. and Ph.D degrees in Electrical Information Engineering from Harbin Engineering University in 2002 and 2006, respectively. She has been a teacher in Harbin Engineering University of China since 2002, and became an associate professor in 2007. During 2007-2008, she stayed in School of Electronics and Computer Science from University of Southampton as a visiting scholar. Now she is a member of China Institute of Communications and a member of China Computer Federation.

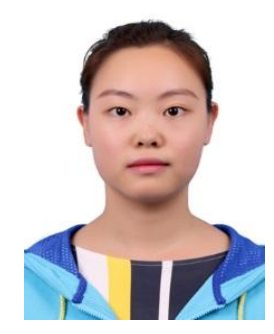

Jing Dai received the B.S. degrees in Northeast Agricultural University in 2015. Now she is studying as a master course student in Electrical Information Engineering from Harbin Engineering University. She study on the resource allocation algorithms and interference suppression.

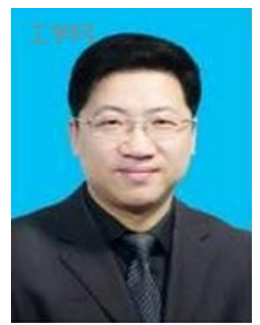

Yibing Li received the B.S., M.S. and Ph.D degrees in Harbin Marine engineering college, Harbin engineering university in 1989, 1997 and 2003, respectively. He has been a teacher in Harbin Engineering University of China since 1989, and became a professor in 2004. During 2007-2008, he stayed in the University of Hong Kong Electronic Engineering lab as a visiting scholar. Now he is a senior member of China Institute of Communications and a senior member of China Computer Federation. 